\title{
Plate-impact-driven ring expansion test (PIDRET) for dynamic fragmentation
}

\author{
Fanny Gant ${ }^{1,2^{*}}$, Gabriel Seisson ${ }^{1}$, Patrice Longère ${ }^{2}$, Skander El Maï ${ }^{1}$ and Jean-Luc \\ Zinszner $^{1}$ \\ ${ }^{1}$ CEA-DAM, Centre de Gramat, 46500 Gramat, France \\ ${ }^{2}$ Institut Clément Ader, Université de Toulouse, ISAE-SUPAERO, MINES ALBI, UPS, INSA, \\ CNRS, Toulouse, France
}

\begin{abstract}
A new experimental set-up mounted at the muzzle of a singlestage gas gun has been designed in order to study the fragmentation of metallic rings under dynamic radial expansion. This concept takes advantage of the quasi-incompressibility of HDPE whose radial flow under plate impact-like loading is used to apply a pressure boundary condition at the ring's inner surface. For the experimental configurations considered in the present work, the average strain rate in the ring reaches values close to $10^{4} \mathrm{~s}^{-1}$. The repeatability and the reliability of the experiments are verified for rings made of steel and aluminium.
\end{abstract}

\section{Introduction}

Understanding the dynamic fragmentation of metallic case under radial expansion is an important issue for industrial and military applications. Indeed, during an accidental vessel's explosion [1] or the aerial detonation of an air-launched warhead, the metallic case inflates until fragmentation occurs generating fragments ejected at high velocities and distances, inducing safety/lethality areas. The definition of these areas needs a good insight in the fragmentation population in terms of mass, number, velocity and angle of ejection. Real-scale experiments are usually performed to obtain these data but, in addition to being very expensive, they are not well suited to investigate the fragmentation phenomenon in details.

Consequently, previous experimental studies generally considered simplified reducedscale configurations such as expansion of a hemispherical shell [2, 3], a cone [4] or a cylinder [5,6]. In [6], a cylinder of high explosive was also used to accelerate several rings simultaneously. Yet, the detonation generates a powerful flash and a cloud of detonation products making difficult to observe the processes of necking and fragmentation. [7, 8] investigated ring expansion driven by electromagnetic force which enabled to satisfyingly observe the ring from homogeneous deformation until rupture. However, the ring underwent heating (Joule effect) that complicated the subsequent analysis. Moreover, during the fragmentation, electric arcs were generated at rupture points that modified the local surface appearance. Then, Zhang et al. [9] used a modified split Hopkinson pressure

\footnotetext{
* Corresponding author: fanny.gant@cea.fr
} 
bar (SHPB) set-up to put water under pressure and expand a ring at strain rate up to $10^{3} \mathrm{~s}^{-1}$. This set-up enabled a good observation of the experiment and was shown repeatable.

In this line, this study presents a new experimental set-up mounted at the muzzle of a single-stage gas gun. The concept takes advantage of the quasi-incompressibility of highdensity polyethylene (HDPE) whose radial flow under plate impact-like loading is used to apply a pressure boundary condition at the ring's inner surface. For the experimental configurations considered in the present work, the average strain rate in the ring is expected to reach values close to $10^{4} \mathrm{~s}^{-1}$. The objective is to verify the repeatability and the reliability of the experiments during a campaign for rings made of steel and aluminium. It ambitions to provide useful and reliable information for theoretical approaches such as those related to linear stability analysis [8, 10] and Mott's approach [11], namely expansion velocity, number of necks, time at the necking onset, and number and mass of fragments. Thus, the ring expansion must be as much axisymmetric and repeatable as possible.

After the presentation of the experimental set-up in Section 2, results obtained on rings made of steel and aluminium are shown and analysed in Section 3. Some concluding remarks and future works are given in Section 4.

\section{Experimental set-up}

\subsection{Principle of the plate impact-driven ring expansion test (PIDRET)}

Fig.1 presents a schematic drawing of the PIDRET set-up part mounted at the gas gun muzzle. The steel impactor projectile (1.2) hits the HDPE disk (2) which is crushed onto the steel anvil (4). In the same time, the disk's material flows radially, inducing a pressure on the inner surface of the ring (3) which radially expands until potential fragmentation. The fragments are ultimately collected in a polymer-foam ring (8), see Fig.2. The 40-mmdiameter ring's square-cross section area is $1 \mathrm{x} 1 \mathrm{~mm}^{2}$.

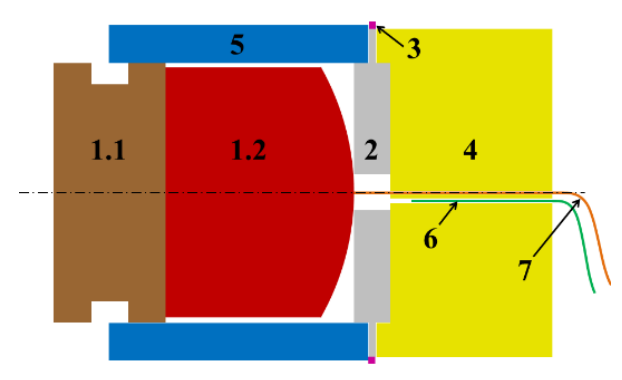

Fig. 1. Schematic drawing of the PIDRET setup. 2D side view near the gas gun muzzle. 1.1: projectile guide. 1.2: impactor projectile. 2: HDPE disk. 3: metal ring. 4: anvil. 5: disk guide. 6: PDV probe. 7: contact sensor.

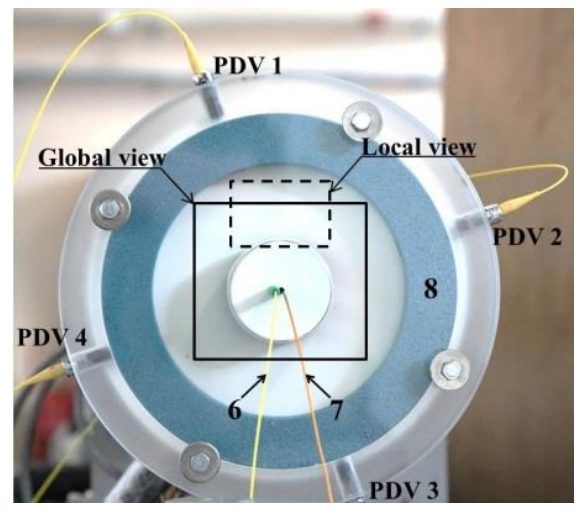

Fig. 2. Diagnostics of the PIDRET set-up. Face view from the gas gun muzzle end. 6: PDV probe. 7: contact sensor. 8: polymer foam ring.

\subsection{Measurement and viewing}

The overall measurement environment is drawn in Fig. 3 and Fig.2 gives a photograph of the gas gun muzzle. The projectile instant of passage and the impact velocity are deduced 


\section{DYMAT 2021}

from the cut-off of three pins inserted into the gas gun's muzzle. A redundant measure of the impact velocity is done by a collimated photon Doppler velocimetry (PDV) probe (6) collinear to the gas gun axis. A contact sensor (7) captures the instant when the projectile hits the HDPE disk. This contact sensor triggers also the video recording of 102 frames by two ultra-high-speed cameras Shimadzu HPV-2. Each camera has its own frame rate and observation window, see Table 1, allowing to get both global and local pictures of the ring expansion. Focalised PDV probes (PDV1-PDV4 in Fig.2), inserted in a support, measure the radial velocity of the ring in four positions, at $90^{\circ}$ from each other.

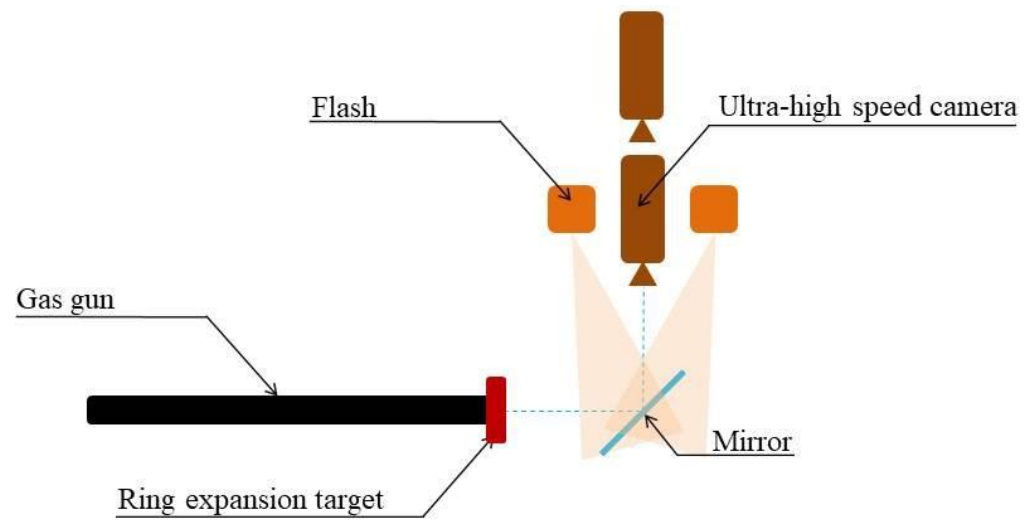

Fig. 3. Schematic drawing of the overall experimental set-up and its measurement environment.

Table 1. Measurement specifications for the global and local views using the two ultra-high-speed cameras Shimadzu HPV-2.

\begin{tabular}{|c||c|c|c|c|}
\hline View & $\begin{array}{c}\text { Observation window } \\
\left(\mathbf{m m}^{2}\right)\end{array}$ & $\begin{array}{c}\text { Spatial resolution } \\
\left.\mathbf{( p i x e l}^{\mathbf{2}}\right)\end{array}$ & $\begin{array}{c}\text { Frame rate } \\
\text { (fps) }\end{array}$ & $\begin{array}{c}\text { Number of } \\
\text { pixels/mm }\end{array}$ \\
\hline Global & $100 \times 80$ & $312 \times 260$ & $250 \mathrm{k}$ & $\sim 3$ \\
\hline Local & $30 \times 25$ & $312 \times 260$ & $500 \mathrm{k}$ & $\sim 10$ \\
\hline
\end{tabular}

\section{Results}

An experimental campaign was conducted in order to verify the feasibility and the repeatability of the loading conditions and results on the one hand and the reliability of the PIDRET set-up on the other hand. For that purpose, tests were carried out at impact velocities close to $150 \mathrm{~m} \cdot \mathrm{s}^{-1}$ on two materials: a mild steel and an aluminium alloy, whose characteristics are summarised in Table 2 along with the experimental configurations.

Table 2. Material parameters of mild steel and aluminium and experimental results of dynamic ring expansion.

\begin{tabular}{|c||c|c|c||c|c|}
\hline $\begin{array}{c}\text { Material of } \\
\text { the ring }\end{array}$ & $\begin{array}{c}\text { Young's } \\
\text { modulus* } \\
\text { (GPa) }\end{array}$ & $\begin{array}{c}\text { Yield } \\
\text { strength* } \\
\text { (MPa) }\end{array}$ & $\begin{array}{c}\text { Ductility* } \\
\mathbf{( \% )}\end{array}$ & $\begin{array}{c}\text { Total strain at } \\
\text { fragmentation** }\end{array}$ & $\begin{array}{c}\text { Number of } \\
\text { fragments** }\end{array}$ \\
\hline Mild steel & $\sim 200$ & $\sim 455$ & $\sim 20$ & 0.25 & 8 \\
\hline Aluminium & $\sim 70$ & $\sim 250$ & $\sim 15$ & 0.15 & 26 \\
\hline
\end{tabular}


Fig. 4 shows an example of some synchronised results obtained from the PIDRET set-up on a mild steel ring: pictures of the dynamically expanding ring coming from the cameras and history of the radial expansion velocity of the outer periphery of the ring coming from the focalised PDV probes. Values of total true hoop strain and strain rate are also specified on the graphs at some particular points.
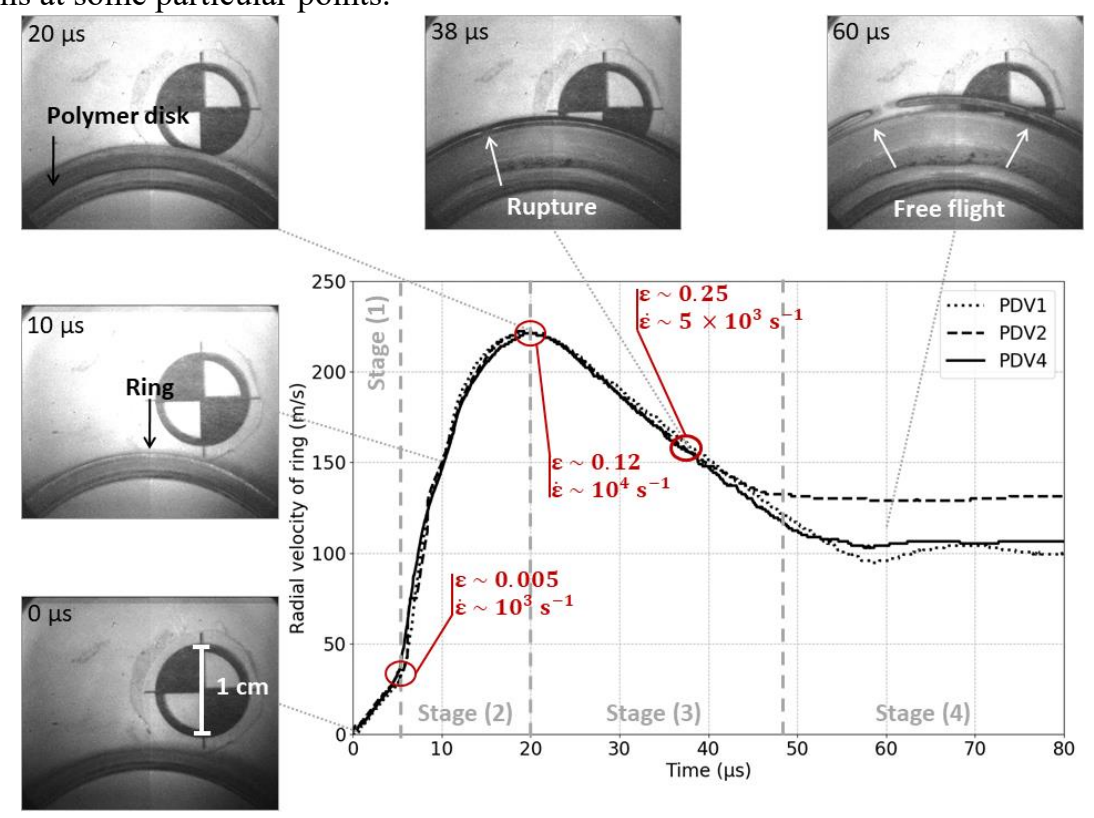

Fig. 4. Expansion of the ring made of mild steel. Impact at $150 \mathrm{~m} \cdot \mathrm{s}^{-1}$. Local view at different instants and history of the radial velocity of 3 points in the periphery of the ring. Global view not displayed here.

Time 0 corresponds to the instant at which the velocity starts rising. The time history of the radial velocity can be decomposed into four stages: (1) linear rising up from 0 to $28 \mathrm{~m} . \mathrm{s}^{-}$ ${ }^{1}$ and lasting about $5 \mu \mathrm{s}$ with a final true hoop strain close to 0.005 , (2) nonlinear rising up from $28 \mathrm{~m} . \mathrm{s}^{-1}$ to $220 \mathrm{~m} . \mathrm{s}^{-1}$ and lasting about $15 \mu \mathrm{s}$ with a final true hoop strain close to 0.12 , (3) quasi linear decrease lasting about $30 \mu \mathrm{s}$, and then (4) plateau. The three radial velocity histories (no result for malfunctioning PDV3) matching very well during stages (1-2) and most of stage (3), one can conclude to a perfectly axisymmetric ring expansion, as expected. During stages (1-3), the true hoop strain rate appears to vary within the range $10^{3}$ $10^{4} \mathrm{~s}^{-1}$.

According to the pictures in Fig.4 (local view), the fragmentation occurs near $38 \mu$ s. Until this instant (at least), the polymer flow seems to keep on exerting a pressure onto the inner surface of the ring. In contrast, the loss of contact (the gap) between the HDPE disk and the ring visible after $60 \mu$ s indicates that the ring, in the form of fragments, is no longer submitted to any external load, thus undergoing a free flight. For this test, the number of fragments is 8 , see Table 2 summarizing the results obtained from the four tests.

By combining the pictures and the velocity plots, the ring seems to homogeneously deform during stages (1-2) and fragmentation occurs during stage (3) for a true hoop strain close to 0.25 , see also Table 2. At this value of strain, the strain rate is close to $5 \times 10^{3} \mathrm{~s}^{-1}$. During stage (4), the fragments are flying freely.

The evolution of the radial velocity shown in Fig. 4 was also observed for the four other tests carried out under the same conditions. One can thus conclude to the feasibility and the 
repeatability of the experimental conditions and results using the developed experimental set-up.

Rings made of aluminium were also tested, see corresponding pictures and superimposition of the radial velocity of both steel and aluminium in Fig.5.
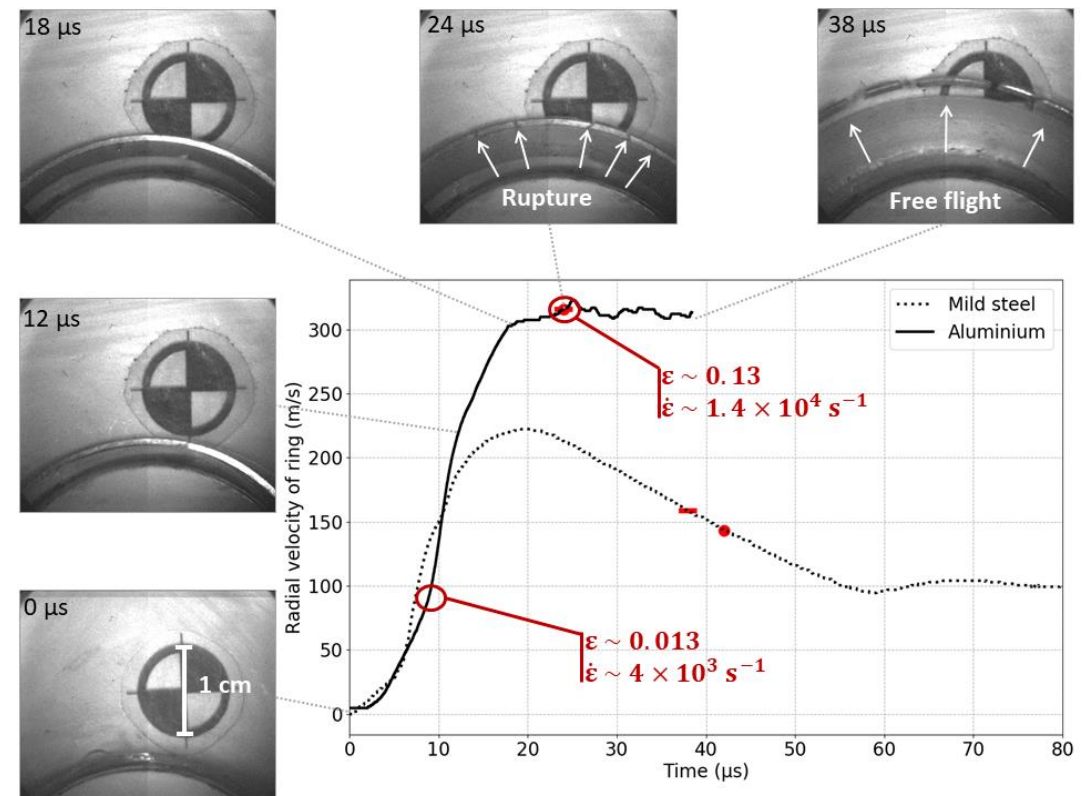

Fig. 5. Radial velocity (PDV1) of mild steel and aluminium rings. Impact at $150 \mathrm{~m} \cdot \mathrm{s}^{-1}$. Local view at different instants for aluminium ring expansion. Dot: fragmentation time identified on the global visualization. Dash: fragmentation time identified on the local visualization.

Contrary to the mild steel, the history of the radial expansion velocity for the aluminium ring may be decomposed into three stages: (1) linear rising up from 0 to $80 \mathrm{~m} . \mathrm{s}^{-1}$ lasting about $8 \mu \mathrm{s}$ with a final true hoop strain close to 0.013 , (2) nonlinear rising up from $80 \mathrm{~m} . \mathrm{s}^{-1}$ to $320 \mathrm{~m} . \mathrm{s}^{-1}$ lasting about $16 \mu \mathrm{s}$ with a final true hoop strain close to 0.13 , and (4) a plateau - stage (3) corresponding to the quasi linear decrease for the steel ring, see above, is not visible for the aluminium ring.

According to the pictures, the aluminium ring seems to homogeneously deform until the fragmentation occurrence at $24 \mu \mathrm{s}$. Before $38 \mu \mathrm{s}$, the HDPE disk is seen to loose contact with the fragments, which consequently fly freely.

The strain rate at the strain at fragmentation, viz. 0.13 (see also Table 2), is close to $1.4 \times 10^{3} \mathrm{~s}^{-1}$. At the maximal radial velocity, which is higher than the mild steel one, the fragmentation occurs immediately generating more fragments (25 fragments for this test) than the mild steel (Table 2). As above mentioned, the quasi-linear decrease of the radial velocity, observed for the mild steel ring expansion, is inhibited by the fracture.

The evolution of the radial velocity of the aluminium ring shown in Fig. 5 was also observed for the four other tests carried out under the same conditions.

\section{Conclusion}

The experimental PIDRET using a single-stage gas gun, presented in this paper, consists in dynamically radially expanding a metallic ring through the polymer flow-induced mechanical loading under impact loading. Diagnostics are used to measure the projectile 
velocity and the radial ring velocity and to video-record the ring expansion. The tests carried out on rings made of steel and aluminium proved the feasibility of the loading conditions on the one hand and the repeatability and reliability of the results on the other hand.

Works are in progress on (i) the improvement of the space and time resolution in order to follow the necking evolution and the fragmentation instant, (ii) the understanding of the phenomena leading to the different stages of the expansion velocity evolution with theoretical and numerical studies, (iii) the potential extension of the tests to other materials.

\section{References}

1. J.W.H. Price. An acetylene cylinder explosion: a most probable cause analysis. Eng. Failure Analysis. 13, 705-715 (2006)

2. C. Bolis, D. Counilh, J.M. Lagrange, P.A. Frugier. Fragmentation of a titanium alloy shell in expansion: from experiment to simulation. Procedia Eng. 58, 672-677 (2013)

3. S. Mercier, N. Granier, A. Molinari, F. Llorca, F. Buy. Multiple necking during the dynamic expansion of hemispherical metallic shells, from experiments to modelling. J. Mecha. Phys. Solids, 58, 955-982 (2010)

4. C. Neel, P. Sable, P. Flater, D. Lacina. Conical impact fragmentation test (CIFT). Int. J. Impact Eng. 140, 1-6 (2020)

5. D.R. Jones, D.J. Chapman, D.E. Eakins. A gas gun based technique for studying the role of temperature in dynamic fracture and fragmentation. J. Applied Phys. 114, 1-10 (2013)

6. D.M. Goto, R. Becker, T.J. Orzechowski, H.K. Springer, A.J. Sunwoo, C.K. Syn. Investigation of the fracture and fragmentation of the explosively driven rings and cylinders. Int. Int. J. Impact Eng. 35, 1547-1556 (2008)

7. H. Zhang, K. Ravi-Chandar. On the dynamics of necking and fragmentation - I. Real time and post-mortem observations in Al 6061-O. Int. J. Fract. 142, 183-217 (2006)

8. S. El Maï. Etude du développement des instabilitées dans un anneau en expansion dynamique. (2014)

9. J. Zhang, F. Zhou, J. Liu. Experimental technique for dynamic fragmentation of liquiddriving expanding ring. EPJ Web Conf. 183, 1-5 (2018)

10. S. Mercier, A. Molinari. Predictions of bifurcation and instabilities during dynamic extension. Int.J. Solids Struct. 40, 1995-2016 (2003)

11. N.F. Mott. Fragmentation of shell cases. Proceeding of the Royal Society. Series A. 300-308 (1945) 